\title{
Enabling citizens to refine the location accuracy of cadastre boundary markers by gamified VGI
}

\author{
Mikko Rönneberga*, Pyry Kettunen ${ }^{\mathrm{a}}$ \\ ${ }^{a}$ Finnish Geospatial Research Institute (FGI, NLS), \{firstname.lastname\}@nls.fi \\ * Corresponding author
}

Keywords: volunteered geographic information, gamification, cadastre, boundary marker, location accuracy

\begin{abstract}
:
National mapping agencies have used the help of citizens in significant data acquisition challenges. The Dutch Kadaster used a mobile application by which border markers of Netherlands and Germany were located and reported by citizens (Olteanu-Raimond 2017). Also, there is a mapping community called the National Map Corps that collects VGI to help the USGS update data about man-made structures in support of the national map and US topographical maps (McCartney et al. 2015). Crowdsourcing is a viable and sometimes the only realistic option when collecting large amounts of spatially distributed data. The selection of a collection method is relevant from both the transparency and the privacy point of view. In geospatial crowdsourcing, there is a clear distinction between contributed geographic information (CGI) and volunteered geographic information (VGI). CGI is collected without the immediate knowledge and consent of the citizen while VGI is inherently voluntary (Bilogrevic 2018). In VGI the citizen is in control of the collection and it is therefore the better choice in terms of transparency. However, this transparency comes with a price as VGI contributions are reliant on motivation of the citizens. Altruism alone is sometimes enough, but other effective methods of motivating citizens to contribute are available. Both utility of gamification, such as points, badges and trophies, and social media utility, such as profiles, ratings and comments, can be used to motivate citizens in crowdsourcing tasks, although different motivation methods apply to different contributor types (Gómez-Barrón et al. 2019).
\end{abstract}

When VGI is used with gamification utility and social media utility, there is a clear need for designing with privacy in mind. Privacy by design paradigm can be used to counter threats of privacy violation, without degrading the quality of the collected data to the point that it is no longer useful (Monreale et al. 2014). As regular citizens are involved instead of experts, the utility-usability tradeoff (Roth 2015) should be leaning towards usability, such as designing an intuitive and simple user interface, rather than focusing on advanced functionality.

This research is conducted following the design science research (DSR) approach. DSR is used to identify a problem, design and create an artifact to solve the problem, demonstrate and evaluate the artifact, and then generalise a solution. As the work in this research is still on-going, this study will feature the problem definition, preliminary research strategy and short high level design description of the artifact solving the identified problem.

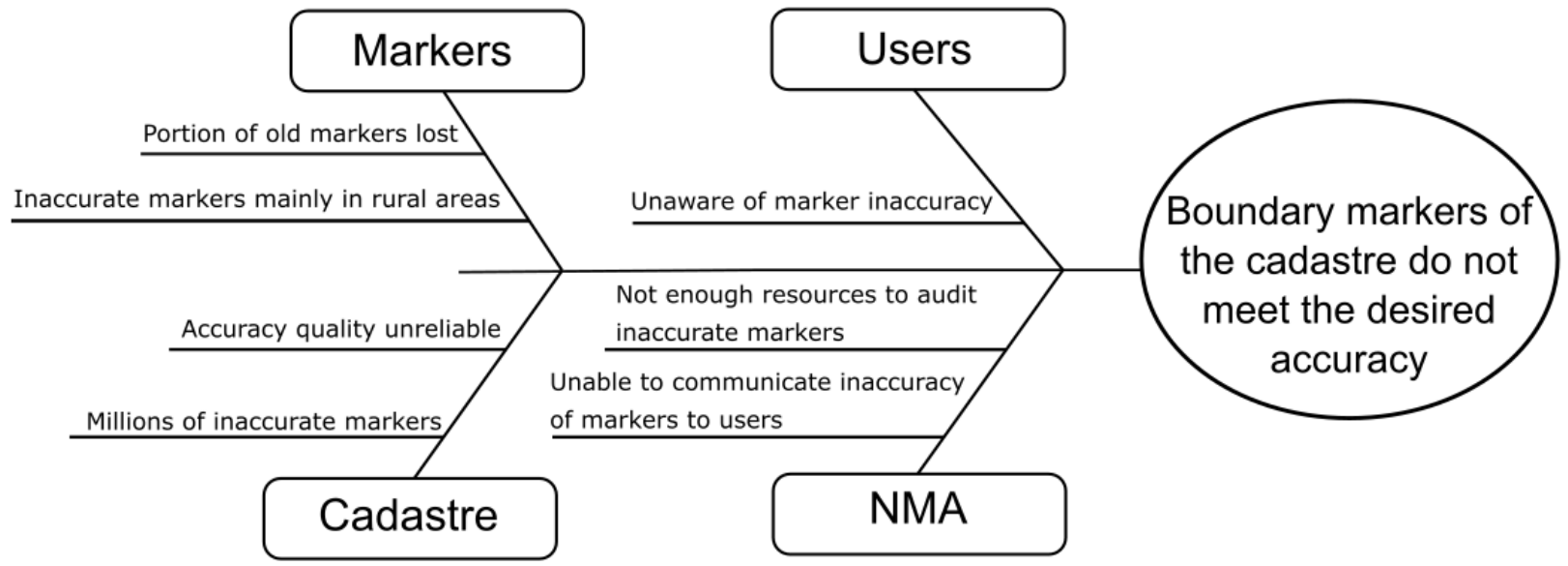

Figure 1. Root causes of why the boundary markers of the cadastre do not meet the desired accuracy.

To begin identifying the problem of why the boundary markers of the cadastre do not meet the desired accuracy, the causal factors of the problem were defined by discussing with national mapping agency (NMA) experts in focus groups and by utilising the root cause analysis process, Figure 1. One root cause of why NMAs face issues with cadastre border markers is their long cadastre history. Many of the border markers can be decades or even a century old and therefore their location accuracy in the cadastre can be meters or even dozens of meters off the mark.. Some markers can be lost 
completely due to vegetation growth or agriculture. Therefore, there is a mismatch between the border marker in the terrain and the digital cadastre. If the mismatch is not or cannot be adequately communicated to the users of the cadastre, the consequences of the cadastre not being accurate can be felt in numerous situations. A simple case can be just that the area of a citizen's real estate used for taxing purposes is inaccurate resulting in additional costs. A more complex case is when a forest cutting machine follows an erroneous borderline of the digital cadastre and cuts a large piece of forest from the neighbouring side. Inaccuracies in the cadastre also result in complaints from the cadastre users. NMAs can audit the cadastre border markers, but this is usually laborious due to the scattered spatial distribution of the inaccurate border markers. The inaccurate markers also tend to be in more difficult to reach rural areas. Audits are often conducted when there is other work to be done on the real estate where the markers lie, but the sheer amount of inaccurate markers in a country wide cadastre can reach millions. This effectively raises both the time and money costs of refining the location accuracy of cadastre markers significantly above what is reasonable to be conducted via NMA audits.

The scope of this study is to enable citizens to refine the border maker accuracy of a national mapping agency cadastre by using a gamified VGI artifact, Figure 2. There are examples of similar endeavors, such as the Dutch Kadaster and USGS National Map Corps, but the gap this study aims to fill is in use of gamified VGI in refining the position accuracy of cadastre border markers. A practical and a more scientific perspective will be explored in this study. First, can VGI be used to refine the location accuracy of cadastre border markers? This research question focuses on the technical capacity of consumer devices, on the quality of the contributed data, such as coverage and accuracy, and on how the gamified VGI approach can be integrated into a NMA production process. Second, are citizens motivated to contribute if gamification methods are applied? This research question focuses on citizens and their motivation to contribute. The motivation effects of the applied gamification methods in the artifact are studied quantitatively and qualitatively.

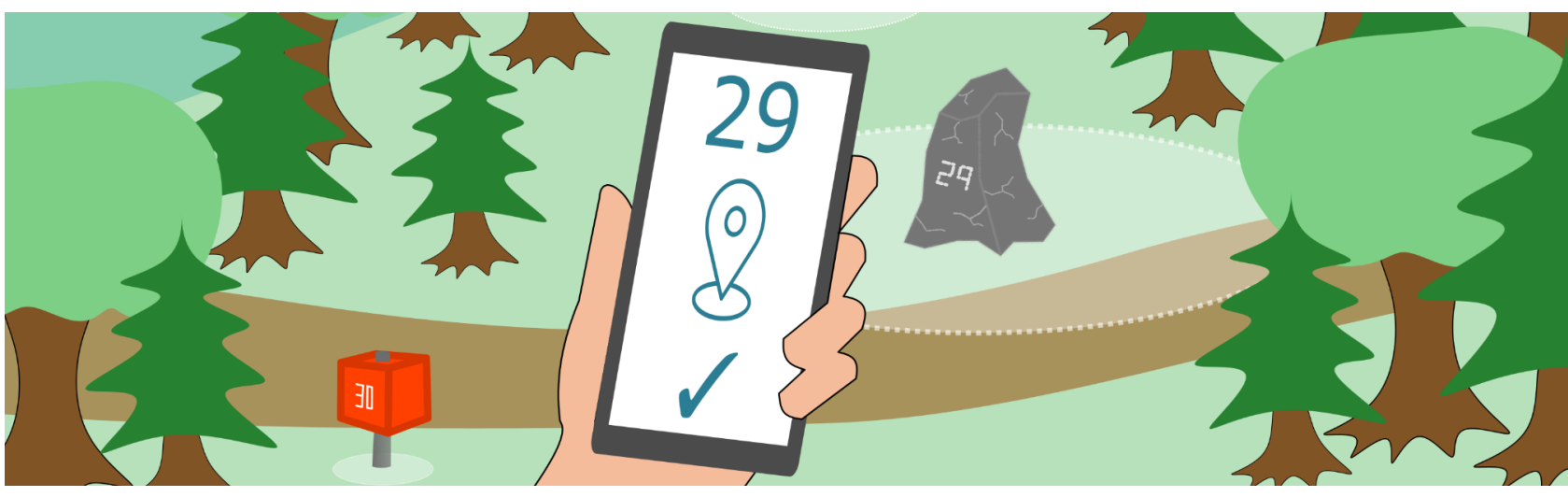

Figure 2. The problem of inaccurate boundary markers is to be solved by designing a mobile application that enables crowdsourced refinement of border marker accuracy.

The research methods planned for evaluation of the created artifact will include data collection from the devices of participants while considering the privacy of the contributors. Think aloud and naturalistic observation is planned to be used during functional tests of the artifact. Questionnaires will be used to gather both quantitative and qualitative data about the artifact. The questionnaires will focus on utility and usability of the artifact. With consent from the citizen the questionnaire will be linked to the artifact user ID to allow for analysis of the questionnaire participants actions using the artifact. The border markers measured by the citizens will be compared to NMA conducted audits to analyse the quality of the contributions. The effect of the applied gamification methods in the artifact are measured based on how much the citizens engage with them. The questionnaire will also be used to evaluate how the applied gamification methods motivated the citizens to contribute.

The artifact is designed for refining the location accuracy of cadastre border markers by utilizing VGI and gamification. The artifact allows participants to locate, measure the position and take pictures of cadastre border markers, and then share their contributions with the NMA. The artifact will be built on web map technology and intended for mobile devices. Privacy of citizens will be taken into account by following the privacy by design approach. Pseudonyms, access control and aggregation are among the methods used. To use the artifact the citizen will need to register with an email address, but this email will not be directly linked to the artifact user profile. Two main aspects of the artifact are measurement corrections of the positioning and gamification of the contribution. The measurement of border markers will utilise a differential global navigation satellite system (DGNSS) to improve the accuracy of the citizen measured border markers. Gamification techniques, such as points, badges and trophies, will be embedded in the border marker measurement process to motivate citizens to contribute.

Future work of this study will include the design, creation and evaluation of the proposed artifact. The solution the artifact offers can also be generalised to other purposes. 